\title{
RESEÑA DE LA PRÁCTICA DEL ESTADO MEXICANO. TRATADOS INTERNACIONALES CELEBRADOS POR MÉXICO, 2001-2002*
}

1. Decreto promulgatorio de las actas finales de la Conferencia Mundial de Radiocomunicaciones (CMR-97), adoptadas en el marco de la Unión Internacional de Telecomunicaciones (UIT), en la ciudad de Ginebra, Suiza, el veintiuno de noviembre de mil novecientos noventa y siete.

Publicación: 13 de julio de 2001.

Categoría: Multilateral.

2. Decreto por el que se aprueba el Convenio de Cooperación en materia de Turismo entre el gobierno de los Estados Unidos Mexicanos y el gobierno de la República de Panamá, firmado en la ciudad de México, el veintidós de mayo de dos mil.

Publicación: 16 de julio de 2001.

3. Decreto promulgatorio entre el gobierno de los Estados Unidos Mexicanos y el gobierno de los Estados Unidos de América en relación al uso de la banda de 2310-2360 mhz, firmado en la ciudad de México, el veinticuatro de julio de 2000.

Publicación: 16 de julio de 2001.

4. Decreto promulgatorio del Acuerdo entre el gobierno de los Estados Unidos Mexicanos y el gobierno del Reino de Suecia, para la Promoción y Protección recíproca de las Inversiones, firmado en la Ciudad de Estocolmo, el tres de octubre de dos mil.

Publicación: 27 de julio de 2001.

5. Acuerdo por el que se da a conocer el Reglamento de Operación del Comité de Integración Regional de Insumos del Tratado de Libre Comercio celebrado entre los Estados Unidos Mexicanos y las repúblicas del Salvador, Guatemala y Honduras, según Acuerdo de la Comisión Administradora del propio tratado.

Publicación: 20 de agosto de 2001.

* El periodo comprendido es el segundo semestre de 2001 y primer semestre de 2002. En esta compilación intervinieron: Leyda Castillo y Dante López Amador. 
6. Decreto promulgatorio del Convenio de Cooperación en materia de Turismo entre el gobierno de los Estados Unidos Mexicanos y el gobierno de la República de Panamá, firmado en la ciudad de México, el veintidós de mayo de 2000.

Publicación: 29 de agosto de 2001.

7. Decreto promulgatorio de la Resolución wha 18.48 Enmiendas al artículo 7o. de la Constitución de la Organización Mundial de la Salud, adoptada en la 18a. Asamblea Mundial de la Salud, durante su decimosegunda sesión plenaria, celebrada el veinte de mayo de mil novecientos sesenta y cinco.

Publicación: 29 de agosto de 2001.

Categoría: multilateral.

8. Decreto promulgatorio de los artículos 24 y 25 de la Constitución de la Organización Mundial de la Salud, adoptada en la 51a. Asamblea Mundial de la Salud, durante su décima sesión plenaria, celebrada el dieciséis de mayo de mil novecientos noventa y ocho.

Publicación: 29 de agosto de 2001.

Categoría: multilateral.

9. Decreto promulgatorio de la Resolución wha 31.18 adopción del texto en árabe y de la reforma del artículo 74 de la Constitución de la Organización Mundial de la Salud, adoptada en la 31a. Asamblea Mundial de la Salud, durante su décima sesión plenaria, celebrada el dieciocho de mayo de mil novecientos setenta y ocho.

Publicación: 29 de agosto de 2001.

Categoría: multilateral.

10. Acuerdo mediante el cual se da a conocer el primer Protocolo Adicional al Acuerdo de Complementación Económica número 51 celebrado entre los Estados Unidos Mexicanos y la República de Cuba. Publicación: 20 de septiembre de 2001.

11. Decreto promulgatorio del Convenio Básico de Cooperación para el Desarrollo entre el gobierno de los Estados Unidos Mexicanos y el gobierno de la República de Cuba, firmado en la Ciudad de la Habana, el doce de abril de mil novecientos noventa y nueve.

Publicación: 21 de septiembre de 2001.

12. Decreto promulgatorio del Convenio Básico de Cooperación Científica y Técnica entre el gobierno de los Estados Unidos Mexicanos y el gobierno de San Kitts y Nevis, firmado en la ciudad de Santo Domin- 
go, República Dominicana, el diecisiete de abril de mil novecientos noventa y nueve.

Publicación: 21 de septiembre de 2001.

13. Decreto promulgatorio del Convenio de Cooperación Educativa y Cultural entre el gobierno de los Estados Unidos Mexicanos y la República de Hungría, suscrito en la Ciudad de Budapest, el veintiséis de octubre de mil novecientos noventa y ocho.

Publicación: 21 de septiembre de 2001.

14. Decreto promulgatorio del Acuerdo sobre Agricultura entre los Estados Unidos Mexicanos y la República de Islandia, firmado en la ciudad de México, el veintisiete de noviembre de 2000.

Publicación: 28 de septiembre de 2001.

15. Acuerdo por el que se da a conocer la entrada en vigor definitiva del Tratado de Libre Comercio entre los Estados Unidos Mexicanos y los Estados de la Asociación Europea de Libre Comercio, para la República de Islandia.

Publicación: 28 de septiembre de 2001.

16. Acuerdo por el que se da a conocer la entrada en vigor definitiva del Tratado de Libre Comercio entre los Estados Unidos Mexicanos y los Estados de la Asociación Europea de Libre Comercio, para el Principado de Liechtenstein.

Publicación: 28 de septiembre de 2001.

17. Acuerdo por el que se da a conocer la entrada en vigor del Tratado de Libre Comercio entre los Estados Unidos Mexicanos y los Estados de la Asociación Europea de Libre Comercio con la República de Islandia y del Acuerdo sobre Agricultura entre los Estados Unidos Mexicanos y la República de Islandia.

Publicación: 28 de septiembre de 2001.

18. Acuerdo relativo a la Salvaguarda Agropecuaria del Tratado de Libre Comercio de América del Norte, mediante el cual se determinan las mercancías (jamón y tocino) comprendidas en las fracciones y con las tasas arancelarias que se indican.

Publicación: 05 de octubre de 2001.

19. Acuerdo relativo a la Salvaguarda Agropecuaria del Tratado de Libre Comercio de América del Norte, mediante el cual se determinan las mercancías (café) comprendidas en las fracciones y con las tasas arancelarias que se indican

Publicación: 05 de octubre de 2001. 
20. Acuerdo por el que se da a conocer la decisión que otorga una dispensa temporal para la utilización de materiales de fuera de la zona de libre comercio para que determinados bienes textiles y del vestido reciban el trato arancelario preferencial del Tratado de Libre Comercio celebrado entre los Estados Unidos Mexicanos, la República de Colombia y la República de Venezuela.

Publicación: 08 de octubre de 2001.

21. Aclaración al Acuerdo por el que se da a conocer la decisión que otorga una dispensa temporal para la utilización de materiales de fuera de la zona de libre comercio para que determinados bienes textiles y del vestido reciban el trato arancelario preferencial del Tratado de Libre Comercio celebrado entre los Estados Unidos Mexicanos, la República de Colombia y la República de Venezuela, publicado el 8 de octubre de 2001. Publicación: 17 de octubre de 2001

22. Decreto para la aplicación del primer Protocolo Adicional al Acuerdo de Complementación Económica núm. 51 suscrito entre el gobierno de los Estados Unidos Mexicanos y el gobierno de la República de Cuba.

Publicación: 24 de octubre de 2001.

23. Decreto promulgatorio del Convenio Básico de Cooperación Técnica y Científica entre el gobierno de los Estados Unidos Mexicanos y el gobierno de Antigua y Barbuda, firmado en la ciudad de Washington, D. C., el veintisiete de julio de mil novecientos noventa y cinco. Publicación: 29 de octubre de 2001.

24. Decreto promulgatorio del Tratado de Cooperación entre el gobierno de los Estados Unidos Mexicanos y el gobierno de la República de Nicaragua sobre asistencia jurídica mutua en materia penal, firmado en la ciudad de Managua, Nicaragua, el diecinueve de diciembre de mil novecientos noventa y siete.

Publicación: 29 de octubre de 2001.

25. Decreto promulgatorio del Acuerdo General de Cooperación entre el gobierno de los Estados Unidos Mexicanos y el gobierno de la República de Sudáfrica, firmado en la ciudad de Nueva York, el veintitrés de septiembre de mil novecientos noventa y ocho.

Publicación: 29 de octubre de 2001.

26. Acuerdo relativo a la Salvaguarda Agropecuaria del Tratado de Libre Comercio de América del Norte, mediante el cual se determinan las 
mercancías comprendidas en las fracciones y con las tasas arancelarias que se indican.

Publicación: 05 de noviembre de 2001

27. Decreto promulgatorio del Convenio Consultivo de la Organización Internacional de Telecomunicaciones Móviles por Satélite en su forma enmendada y de la enmienda del Acuerdo de Explotación de dicha Organización, adoptados en la ciudad de Londres, el veinticuatro de abril de mil novecientos noventa y ocho.

Publicación: 12 de noviembre de 2001

28. Decreto promulgatorio del Convenio Básico de Cooperación Técnica y Científica entre el gobierno de los Estados Unidos Mexicanos y el gobierno de la República de Guatemala, firmado en la ciudad de Guatemala, el veinte de febrero de mil novecientos noventa y ocho.

Publicación: 12 de noviembre de 2001.

29. Decreto por el que se aprueba el Convenio Internacional para la Conservación del Atún del Atlántico, adoptado en Río de Janeiro, el catorce de mayo de mil novecientos sesenta y seis.

Publicación: 16 de noviembre de 2001.

30. Decreto promulgatorio del Convenio entre el gobierno de los Estados Unidos Mexicanos y el gobierno de Rumania en materia de educación, cultura, juventud y deporte, firmado en la cuidad de Bucarest, el veintidós de octubre de mil novecientos noventa y nueve.

Publicación: 7 de diciembre de 2001.

31. Acuerdo relativo a la Salvaguarda Agropecuaria del Tratado de Libre Comercio de América del Norte, mediante el cual se determinan las mercancías comprendidas en las fracciones y con las tasas arancelarias que se indican.

Publicación: 7 de diciembre de 2001.

32. Decreto por el que se aprueba la Constitución de la Organización Internacional para las Migraciones, que entró en vigor el treinta de noviembre de mil novecientos cincuenta y cuatro.

Publicación: 16 de enero de 2002.

Categoría: multilateral

33. Decreto por el que se aprueba la adhesión al Protocolo de firma facultativa sobre jurisdicción obligatoria para la solución de controversias de la Convención de Viena sobre relaciones consulares, adoptado en Viena, el veinticuatro de abril de mil novecientos sesenta y tres. 
Publicación: 16 de enero de 2002

Categoría: multilateral.

34. Decreto por el que se aprueba el retiro parcial de la reserva que el gobierno de México formuló al artículo 25 inciso b) del Pacto Internacional de Derechos Civiles y Políticos, al proceder el depósito de su instrumento de adhesión en marzo de mil novecientos ochenta y uno.

Publicación: 16 de enero de 2002.

Categoría: multilateral.

35. Decreto por el que se aprueba la adhesión al Protocolo facultativo del Pacto Internacional de Derechos Civiles y Políticos, adoptado por la Asamblea General de las Naciones Unidas el dieciséis de diciembre de mil novecientos setenta y seis.

Publicación: 16 de enero de 2002.

Categoría: multilateral.

36. Decreto por el que se aprueba el protocolo facultativo de la Convención sobre los Derechos del Niño relativo a la venta de niños, la prostitución infantil y la utilización de los niños en la pornografía, adoptado por la Asamblea General de las Naciones Unidas el veinticinco de mayo de dos mil.

Publicación: 16 de enero de 2002.

Categoría: multilateral.

37. Decreto por el que se aprueba la Convención sobre la Imprescriptibilidad de los Crímenes de Guerra y de los Crímenes de Lesa Humanidad, adoptada por la Asamblea General de las Naciones Unidas el veintiséis de noviembre de mil novecientos sesenta y ocho.

Publicación: 16 de enero de 2002.

Categoría: multilateral.

38. Decreto por el que se aprueban las enmiendas a los artículos 17 párrafo 7 y 18 párrafo 5 de Convención contra la Tortura y otros Tratos o Penas Crueles, Inhumanos o Degradantes del diez de diciembre de mil novecientos ochenta y cuatro, adoptada en Nueva York, el ocho de septiembre de mil novecientos noventa y dos.

Publicación: 17 de enero de 202

Categoría: multilateral.

39. Decreto por el que se aprueba el Acuerdo entre los Estados Unidos Mexicanos y la República de Cuba para la Promoción y Protección 
recíproca de las Inversiones, firmado en la ciudad de México, el treinta de mayo de dos mil uno.

Publicación: 17 de enero de 2002.

40. Decreto por el que se aprueba la Declaración para el reconocimiento de la competencia del Comité contra la Tortura, de la Convención contra la Tortura y otros Tratos o Penas Crueles, Inhumanos o Degradantes, adoptada por la Asamblea General de las Naciones Unidas el diez de diciembre de mil novecientos ochenta y cuatro.

Publicación: 17 de enero de 2002.

Categoría: multilateral.

41. Decreto por el que se aprueba la Declaración para el reconocimiento de la competencia del Comité para la Eliminación de la Discriminación Racial, de la Convención Internacional sobre la Eliminación de todas las Formas de Discriminación Racial, adoptada por la Asamblea General de las Naciones Unidas el veintiuno de diciembre de mil novecientos sesenta y cinco.

Publicación: 17 de enero de 2002.

Categoría: multilateral.

42. Decreto por el que se aprueba el Protocolo facultativo de la Convención sobre los Derechos del Niño relativo a la participación de niños en los conflictos armados, adoptado por la Asamblea General de las Naciones Unidas el veinticinco de mayo de dos mil.

Publicación: 17 de enero de 2002.

Categoría: multilateral.

43. Decreto por el que se aprueba el retiro parcial de las declaraciones interpretativas y de la reserva que el gobierno de México formuló al párrafo 3 del artículo 12 y al párrafo 2 del artículo 23 respectivamente de la Convención Americana sobre Derechos Humanos, al proceder al depósito de su instrumento de adhesión el veinticuatro de marzo de mil novecientos ochenta y uno.

Publicación: 17 de enero de 2002.

Categoría: multilateral.

44. Decreto por el que se aprueba el Protocolo Facultativo de la Convención sobre la Eliminación de todas las Formas de Discriminación contra la Mujer, adoptado por la Asamblea General de las Naciones Unidas el seis de octubre de mil novecientos noventa y nueve.

Publicación: 18 de enero de 2002.

Categoría: multilateral. 
45. Decreto por el que se aprueba el Protocolo sobre privilegios e inmunidades de la Asociación de Estados del Caribe, adoptado en la Ciudad de Panamá, el trece de diciembre de mil novecientos noventa y nueve.

Publicación: 18 de enero de 2002.

Categoría: multilateral.

46. Decreto por el que se aprueba la Convención Interamericana sobre Desaparición Forzada de Personas, adoptada en la ciudad de Belém, Brasil, el nueve de junio de mil novecientos noventa y cuatro.

Publicación: 18 de enero de 2002.

Categoría: multilateral.

47. Decreto promulgatorio del Convenio entre los Estados Unidos Mexicanos y el Gran Ducado de Luxemburgo para evitar la Doble Imposición y Prevenir la Evasión Fiscal en materia de Impuestos sobre la Renta y sobre el Capital, firmado en la ciudad de Luxemburgo, el siete de febrero de dos mil uno.

Publicación: 6 de febrero de 2002.

48. Fe de erratas al Decreto por el que se aprueba la Convención sobre la Imprescriptibilidad de los Crímenes de Guerra y de los Crímenes de Lesa Humanidad, adoptada por la Asamblea General de las Naciones Unidas el veintiséis de noviembre de mil novecientos setenta y ocho, publicado el 16 de enero de 2002.

Publicación: 11 de febrero de 2002.

Categoría: multilateral.

49. Fe de erratas al Decreto por el que se aprueba la Convención Interamericana sobre Desaparición Forzada de Personas, adoptada en la ciudad de Belém, Brasil, el nueve de junio de mil novecientos noventa y cuatro, publicado el 18 de enero de 2002.

Publicación: 27 de febrero de 2002.

50. Acuerdo relativo a la Salvaguarda Agropecuaria del Tratado de Libre Comercio de América del norte, mediante el cual se determinan las mercancías comprendidas en las fracciones y con las tasas arancelarias que se indican (Estados Unidos de América).

Publicación: 01 de marzo de 2002.

51. Acuerdo relativo a la Salvaguarda Agropecuaria del Tratado de Libre Comercio de América del Norte, mediante el cual se determinan las mercancías comprendidas en las fracciones y con las tasas arancelarias que se indican (Canadá).

Publicación: 01 de marzo de 2002. 
52. Decreto promulgatorio del Tratado de la OMPI sobre Derecho de Autor, adoptado en el marco de la Conferencia Diplomática sobre ciertas cuestiones de derecho de autor y derechos conexos de la Organización Mundial de la Propiedad Intelectual, celebrada en la ciudad de Ginebra, Suiza, el veinte de diciembre de mil novecientos noventa y seis.

Publicación: 15 de marzo de 2002.

Categoría: multilateral.

53. Acuerdo relativo a la Salvaguarda Agropecuaria del Tratado de Libre Comercio de América del Norte, mediante el cual se determinan las mercancías comprendidas en las fracciones y con las tasas arancelarias que se indican (Canadá).

Publicación: 09 de abril de 2002.

54. Acuerdo relativo a la Salvaguarda Agropecuaria del Tratado de Libre Comercio de América del Norte, mediante el cual se determinan las mercancías comprendidas en las fracciones y con las tasas arancelarias que se indican (Estados Unidos de América).

Publicación: 09 de abril de 2002.

55. Acuerdo relativo a la Salvaguarda Agropecuaria del Tratado de Libre Comercio de América del norte, mediante el cual se determina la mercancía comprendida en la fracción y con la tasa arancelaria que se indica.

Publicación: 18 de abril de 2002.

56. Decreto promulgatorio del protocolo facultativo de la Convención sobre los Derechos del Niño relativo a la venta de niños, la prostitución infantil y la utilización de los niños en la pornografía, adoptado por la Asamblea General de las Naciones Unidas el veinticinco de mayo de dos mil.

Publicación: 22 de abril de 2002.

Categoría: multilateral.

57. Decreto promulgatorio de la Convención sobre Imprescriptibilidad de los Crímenes de Guerra y de los Crímenes de Lesa Humanidad, adoptada por la Asamblea General de las Naciones Unidas el veintiséis de noviembre de mil novecientos sesenta y ocho.

Publicación: 22 de abril de 2002.

Categoría: multilateral.

58. Decreto promulgatorio del Convenio sobre Readaptación Profesional y el Empleo de Personas Inválidas, adoptado por la conferencia general de la Organización Internacional del trabajo, durante la sexagési- 
ma novena reunión, celebrada en Ginebra, el veinte de junio de mil novecientos ochenta y tres.

Publicación: 22 de abril de 2002.

Categoría: multilateral.

59. Decreto promulgatorio del Convenio de Reconocimiento Mutuo de Certificados de Estudios, Títulos y Grados Académicos de Educación Superior entre el gobierno de los Estados Unidos Mexicanos y el gobierno de la República de Colombia, firmado en la ciudad de México, el siete de diciembre de mil novecientos noventa y ocho.

Publicación: 22 de abril de 2002.

60. Decreto por el que se aprueba el Acuerdo de sede entre los Estados Unidos Mexicanos y el Banco de Pagos Internacionales en relación con el establecimiento y estatuto de una oficina de representación del Banco de Pagos Internacionales en México, firmado en la ciudad de México, el cinco de noviembre de dos mil uno.

Publicación: 26 de abril de 2002.

Categoría: multilateral.

61. Decreto por el que se aprueba el Acuerdo entre los Estados Unidos Mexicanos y el Comité Internacional de la Cruz Roja relativo al establecimiento en México de una delegación regional del comité, firmado en la ciudad de México, el veinte de julio de dos mil uno.

Publicación: 02 de mayo de 2002.

Categoría: multilateral.

62. Acuerdo relativo a la Salvaguarda Agropecuaria del Tratado de Libre Comercio de América del Norte, mediante el cual se determina la mercancía comprendida en la fracción y con la tasa arancelaria que se indica.

Publicación: 02 de mayo de 2002.

63. Decreto promulgatorio del protocolo facultativo de la Convención sobre la Eliminación de todas las Formas de Discriminación contra la Mujer, adoptado por la Asamblea General de las Naciones Unidas el seis de octubre de mil novecientos noventa y nueve.

Publicación: 03 de mayo de 2002.

Categoría: multilateral.

64. Decreto promulgatorio de las enmiendas a los artículos 17 párrafo 7 y 18 párrafo 5 de la Convención contra la Tortura y otros Tratos o Penas Crueles, Inhumanos o Degradantes del diez de diciembre de mil 
novecientos ochenta y cuatro, adoptadas en Nueva York, el ocho de septiembre de mil novecientos noventa y dos.

Publicación: 03 de mayo de 2002.

Categoría: multilateral.

65. Decreto promulgatorio de la declaración para el reconocimiento de la competencia del Comité contra la Tortura, de la Convención contra la Tortura y otros Tratos o Penas Crueles, Inhumanos o Degradantes, adoptada por la Asamblea General de las Naciones Unidas, el diez de diciembre de mil novecientos ochenta y cuatro.

Publicación: 03 de mayo de 2002.

66. Decreto promulgatorio de la declaración para el reconocimiento de la competencia del Comité para la Eliminación de la Discriminación Racial, de la Convención Internacional sobre la Eliminación de todas las Formas de Discriminación Racial, adoptada por la Asamblea General de las Naciones Unidas el veintiuno de diciembre de mil novecientos sesenta y cinco.

Publicación: 03 de mayo de 2002.

Categoría: multilateral.

67. Decreto promulgatorio del protocolo facultativo de la Convención sobre los Derechos del Niño relativo a la Participación de Niños en los Conflictos Armados, adoptado por la Asamblea General de las Naciones Unidas el veinticinco de mayo de dos mil.

Publicación: 03 de mayo de 2002.

Categoría: multilateral.

68. Decreto promulgatorio de la adhesión al Protocolo de firma facultativa sobre jurisdicción obligatoria para la solución de controversias de la Convención de Viena sobre Relaciones Consulares, adoptado en Viena el veinticuatro de abril de mil novecientos sesenta y tres.

Publicación: 03 de mayo de 2002.

Categoría: multilateral.

69. Decreto promulgatorio del Acuerdo entre los Estados Unidos Mexicanos y la República de Cuba para la Promoción y Protección recíproca de las Inversiones, firmado en la ciudad de México, el treinta de mayo de dos mil uno.

Publicación: 03 de mayo de 2002.

70. Decreto promulgatorio de la adhesión al protocolo facultativo del Pacto Internacional de Derechos Civiles y Políticos, adoptado por la 
Asamblea General de las Naciones Unidas el dieciséis de diciembre de mil novecientos sesenta y seis.

Publicación: 03 de mayo de 2002.

71. Decreto promulgatorio de la Convención Interamericana sobre Desaparición Forzada de Personas, adoptada en la ciudad de Belém, Brasil, el nueve de junio de mil novecientos noventa y cuatro.

Publicación: 06 de mayo de 2002.

72. Decreto por el que se aprueba el Acuerdo entre el gobierno de los Estados Unidos Mexicanos y el gobierno de la República de Corea para la Promoción y Protección recíproca de las Inversiones, firmado en la ciudad de Bandar Seri Begawan, Brunei Darussalam, el catorce de noviembre de dos mil.

Publicación: 24 de mayo de 2002.

73. Decreto por el que se aprueba el Convenio entre el gobierno de los Estados Unidos Mexicanos y el gobierno de la República Checa sobre Cooperación Educativa y Cultural, firmado en la ciudad de Praga, el once de octubre de dos mil uno.

Publicación: 24 de mayo de 2002.

74. Decreto por el que se aprueba el Convenio entre el gobierno de los Estados Unidos Mexicanos y el gobierno de la República de Lituania sobre cooperación en los campos de la educación, la cultura, el arte y el deporte, firmado en la ciudad de México, el veinticuatro de enero de dos mil dos.

Publicación: 24 de mayo de 2002.

75. Decreto por el que se aprueba el Acuerdo de Cooperación entre el gobierno de los Estados Unidos Mexicanos y el gobierno de la República Italiana en materia de Lucha contra el Crimen Organizado, firmado en la ciudad de México, el diecinueve de noviembre de dos mil uno. Publicación: 24 de mayo de 2002.

76. Decreto por el que se aprueba la adhesión al Convenio Internacional del Café de 2001, adoptado mediante resolución número 393 del consejo Internacional del café, el 28 de septiembre de 2000.

Publicación: 24 de mayo de 2002.

Categoría: multilateral

77. Decreto promulgatorio del Acuerdo entre los Estados Unidos Mexicanos y el Comité Internacional de la Cruz Roja relativo al establecimiento en México de una delegación regional del comité, firmado en la ciudad de México, el veinte de julio de dos mil uno. 
Publicación: 24 de mayo de 2002.

Categoría: multilateral.

78. Decreto promulgatorio del Tratado de la OMPI sobre Interpretación o Ejecución y Fonogramas, adoptado en el marco de la conferencia diplomática sobre ciertas cuestiones de derecho de autor y derechos conexos de la Organización Mundial de la Propiedad Intelectual, celebrada en la ciudad de Ginebra, Suiza, el veinte de diciembre de mil novecientos noventa y seis.

Publicación: 27 de mayo de 2002.

Categoría: multilateral.

79. Decreto promulgatorio del Convenio relativo a la Organización Hidrográfica Internacional, adoptado en la Ciudad de Mónaco, el tres de mayo de mil novecientos sesenta y siete.

Publicación: 03 de junio de 2002.

Categoría: multilateral.

80. Decreto promulgatorio del Acuerdo de sede entre los Estados Unidos Mexicanos y el Banco de Pagos Internacionales en relación con el establecimiento y Estatuto de una oficina de representación del Banco de Pagos Internacionales en México, firmado en la ciudad de México, el cinco de noviembre de dos mil uno.

Publicación: 20 de junio de 2002.

81. Decreto promulgatorio del Convenio entre el gobierno de los Estados Unidos Mexicanos y el gobierno de la República Checa sobre Cooperación Educativa y Cultural, firmado en la Ciudad de Praga, el once de octubre de dos mil uno.

Publicación: 27 de junio de 2002.

82. Decreto por el que se aprueba el Protocolo de Cartagena sobre Seguridad de la Biotecnología del Convenio sobre Diversidad Biológica, adoptado en Montreal, el veintinueve de enero de dos mil.

Publicación: 01 de julio de 2002.

83. Decreto promulgatorio del Convenio Internacional para la Conservación del Atún del Atlántico, adoptado en la Ciudad de Río de Janeiro, Brasil, el catorce de mayo de mil novecientos sesenta y seis.

Publicación: 17 de julio de 2002. 